\title{
LAURENT COEFFICIENTS AND EXT OF FINITE GRADED MODULES
}

\author{
Luchezar L. Avramov \\ RAGNAR-OlaF BuchWEITZ \\ JUDITH D. SALLY
}

Let $R=\bigoplus_{n \geqslant 0} R_{n}$ be a graded commutative ring generated over a field $K=R_{0}$ by homogeneous elements $x_{1}, \ldots, x_{e}$ of positive degrees $d_{1}, \ldots, d_{e}$. The Hilbert-Serre Theorem shows that for each finite graded $R$-module $M=\bigoplus_{n \in \mathbb{Z}} M_{n}$ the Hilbert series $\sum_{n \in \mathbb{Z}}\left(\operatorname{rank}_{K} M_{n}\right) t^{n}$ is the Laurent expansion around 0 of a rational function

$$
H_{M}(t)=\frac{q_{M}(t)}{\prod_{i=1}^{e}\left(1-t^{d_{i}}\right)}
$$

with $q_{M}(t) \in \mathbb{Z}\left[t, t^{-1}\right]$. We demonstrate that Laurent expansions $[M]_{z}$ of $H_{M}(t)$ around other points $z$ of the extended complex plane $\overline{\mathbb{C}}$ also carry important structural information.

When $R$ is generated in degree one and $z=1$ such an approach has been used implicitly, since the coefficients of the principal part of $[M]_{1}$ are then also coefficients of the Hilbert polynomial of $M$. Without assumptions on the generation of $R$, coefficients of $[M]_{1}$ have been sighted occasionally in invariant theory. In that context Benson and Crawley-Boevey [3] have discovered recently that for all finite graded modules over a graded normal domain the coefficient $\psi$ of $1 /(1-t)^{\operatorname{dim} R-1}$ satisfies an equality

$$
\begin{gathered}
\psi\left(\operatorname{Hom}_{R}(M, N)\right)-\psi\left(\operatorname{Ext}_{R}^{1}(M, N)\right)= \\
\operatorname{rank}_{R}(M) \operatorname{rank}_{R}(N) \psi(R)+\operatorname{rank}_{R}(M) \psi(N)-\operatorname{rank}_{R}(N) \psi(M) .
\end{gathered}
$$

The present investigation started as an attempt to "explain" this intriguing formula, and to find out whether analogous equalities exist for subsequent coefficients of Laurent expansions around 1. Fairly complete answers are presented in Sections 3 and 4. They depend on embedding (1) into an equality of Laurent series, which "predicts" an infinite sequence of similar relations among higher Laurent coefficients. The results are proved in Section 1, and are used in Section 2 to obtain non-trivial lower bounds for the Bass numbers of certain graded modules of finite injective dimension. 


\section{HilberT SERIES}

When $M$ and $N$ are graded $R$-modules $\operatorname{Hom}_{R}(M, N)_{n}$ stands for the $K$-vector subspace of $\operatorname{Hom}_{R}(M, N)$ consisting of homomorphisms $\alpha$ of degree $n$, that is, such that $\alpha\left(M_{j}\right) \subseteq$ $N_{n+j}$ for $j \in \mathbb{Z}$. For a finite $M$ the inclusion $\bigoplus_{n \in \mathbb{Z}} \operatorname{Hom}_{R}(M, N)_{n} \subseteq \operatorname{Hom}_{R}(M, N)$ is an equality, and thus $\operatorname{Hom}_{R}(M, N)$ is graded; as $M$ has a resolution by finite free graded $R$ modules and homomorphisms of degree 0, this induces for each $i$ a grading on $\operatorname{Ext}_{R}^{i}(M, N)$.

Theorem 1. If $M$ and $N$ are finite graded $R$-modules such that $\operatorname{Ext}_{R}^{i}(M, N)=0$ for $i \gg 0$, then there is an equality of rational functions

$$
\sum_{i}(-1)^{i} H_{\operatorname{Ext}_{R}^{i}(M, N)}(t)=\frac{H_{M}\left(t^{-1}\right) \cdot H_{N}(t)}{H_{R}\left(t^{-1}\right)} .
$$

Remark. This is a far reaching generalization of the known expression $H_{\operatorname{Ext}_{R}^{d-m}(M, \omega)}(t)=$ $(-1)^{m} H_{M}\left(t^{-1}\right)$ which holds for an $m$-dimensional graded Cohen-Macaulay module $M$ over a $d$-dimensional Cohen-Macaulay ring $R$ with canonical module $\omega$, cf. [4; (4.3.7)].

Indeed, $\operatorname{Ext}_{R}^{i}(M, \omega)=0$ unless $i=d-m$ by [4; (3.3.10)], so we only need to show that $H_{\omega}(t)=(-1)^{d} H_{R}\left(t^{-1}\right)$. Consider the polynomial ring $Q=K\left[X_{1}, \ldots, X_{e}\right]$ graded by $\operatorname{deg} X_{j}=d_{j}$ and the surjective homomorphism of $K$-algebras $Q \rightarrow R$ with $X_{j} \mapsto x_{j}$ for $1 \leq j \leq e$. By $[4 ;(3.6 .12),(3.6 .10)]$, for $W=Q\left(-\sum_{i=1}^{e} d_{i}\right)$ we have $\omega \cong \operatorname{Ext}_{Q}^{e-d}(R, W)$, hence we get $H_{\omega}(t)$ by applying the theorem to the $Q$-modules $R$ and $W$.

When $f(t)$ is a function of a complex variable $t$, we denote by $[f(t)]_{z}$ the expansion of $f(t)$ as a Laurent series $\sum_{j \in \mathbb{Z}} a_{j}(t-z)^{j}$ when $z \in \mathbb{C}$, and as a Laurent series $\sum_{j \in \mathbb{Z}} a_{j} t^{-j}$ when $z=\infty$. The order of $[f(t)]_{z}$ is introduced by ord $[f(t)]_{z}=\inf \left\{j \in \mathbb{Z} \mid a_{j} \neq 0\right\}$.

For a finite graded $R$-module $M$ we often write $[M]_{z}$ in place of $\left[H_{M}(t)\right]_{z}$. Clearly, $[M]_{0} \in \mathbb{Z}((t))$ and $[M]_{\infty} \in \mathbb{Z}\left(\left(t^{-1}\right)\right)$, where $\mathbb{A}((t))=\mathbb{A}[[t]]\left[t^{-1}\right]$ and $\mathbb{A}\left(\left(t^{-1}\right)\right)=\mathbb{A}\left[\left[t^{-1}\right]\right][t]$ denote rings of formal Laurent series of finite order with coefficients in $\mathbb{A}$, with topologies defined by the powers of the respective indeterminate. Note that if $\left\{g_{i}\right\}_{i \geqslant 0}$ is a sequence of formal Laurent series in either ring, then $\sum_{i \geqslant 0} g_{i}$ converges when $\lim _{i \rightarrow \infty}$ ord $g_{i}=\infty$.

Much of the paper deals with properties of the rational function

$$
\phi_{R}(M, N)(t)=\frac{H_{M}\left(t^{-1}\right) \cdot H_{N}(t)}{H_{R}\left(t^{-1}\right)} .
$$

Without homological assumptions on $M$ or $N$, we establish the following limited

Proposition 2. If $M$ and $N$ are finite graded $R$-modules, and $N$ has finite length, then $H_{\mathrm{Ext}_{R}^{i}(M, N)}(t) \in \mathbb{Z}\left[t, t^{-1}\right]$, the order of $\left[\operatorname{Ext}_{R}^{i}(M, N)\right]_{\infty}$ goes to infinity with $i$, and $\sum_{i}(-1)^{i}\left[\operatorname{Ext}_{R}^{i}(M, N)\right.$ $\mathbb{Z}\left(\left(t^{-1}\right)\right)$ is the Laurent expansion around $\infty$ of $\phi_{R}(M, N)(t)$.

For comparison and for later application, we recall the situation in homology. The grading of $M \otimes_{K} N$ by $\left(M \otimes_{K} N\right)_{n}=\bigoplus_{j \in \mathbb{Z}}\left(M_{j} \otimes_{K} N_{n-j}\right)$ induces a grading of $M \otimes_{R} N$. As above, this defines a grading of $\operatorname{Tor}_{i}^{R}(M, N)$ for each $i$, and by [1; Lemma 7] we have

Lemma 3. If $M$ and $N$ are finite graded $R$-modules, then the order of $\left[\operatorname{Tor}_{i}^{R}(M, N)\right]_{0} \in$ $\mathbb{Z}((t))$ goes to $\infty$ with $i$, and $\sum_{i}(-1)^{i}\left[\operatorname{Tor}_{i}^{R}(M, N)\right]_{0} \in \mathbb{Z}((t))$ is the Laurent expansion around 0 of the rational function

$$
\chi^{R}(M, N)(t)=\frac{H_{M}(t) \cdot H_{N}(t)}{s T} .
$$


Before starting on the proofs we record some general properties of Laurent expansions. Remark. If $0 \rightarrow M^{\prime} \rightarrow M \rightarrow M^{\prime \prime} \rightarrow 0$ is an exact sequence of graded $R$-modules, then $\left[M^{\prime}\right]_{z}+\left[M^{\prime \prime}\right]_{z}=[M]_{z}$. Indeed, additivity is obvious for $z=0$, hence yields an equality of rational functions $H_{M^{\prime}}(t)+H_{M^{\prime \prime}}(t)=H_{M}(t)$, which implies additivity for arbitrary $z$.

Similarly, one sees that $[M(a)]_{z}=\left[t^{-a}\right]_{z}[M]_{z}$ for any $a \in \mathbb{Z}$, where $M(a)$ is the $a$ 'th translate of $M$, that is, the graded $R$-module with $M(a)_{n}=M_{a+n}$ for $n \in \mathbb{Z}$.

Proof of Theorem 1. Let $Q \rightarrow R$ and $W$ be as in the remark following the statement of the theorem, let $I^{\bullet}$ be a minimal (hence finite, cf. [4, (3.6.6)]) graded injective resolution of the $Q$-module $W$, and let $F^{\bullet}$ be a minimal graded free resolution of the $R$-module $M$. As in $[2,(4.4 . \mathrm{I})]$, there is a canonical isomorphism of complexes of graded $R$-modules

$$
\theta: F^{\bullet} \otimes_{R} \operatorname{Hom}_{Q}\left(N, I^{\bullet}\right) \rightarrow \operatorname{Hom}_{Q}\left(\operatorname{Hom}_{R}\left(F^{\bullet}, N\right), I^{\bullet}\right)
$$

given by $\theta\left(f \otimes_{R} \alpha\right)(\beta)=(-1)^{i(j-h)} \alpha \beta(f)$ for $f \in F^{i}, \alpha \in \operatorname{Hom}_{Q}\left(N, I^{j}\right), \beta \in \operatorname{Hom}_{Q}\left(F^{h}, N\right)$. Filtering the complex on the left by the cohomological degree of $F^{\bullet}$, and the one on the right by that of $I^{\bullet}$, we obtain two spectral sequences which converge to a common limit:

$$
{ }_{2} \mathrm{E}_{p}^{q}=\operatorname{Tor}_{p}^{R}\left(M, \operatorname{Ext}_{Q}^{q}(N, W)\right) \Longrightarrow E_{p-q} \Longleftarrow \operatorname{Ext}_{Q}^{q}\left(\operatorname{Ext}_{R}^{p}(M, N), W\right)={ }^{2} \mathrm{E}^{p, q} .
$$

In the first spectral sequence we have ${ }_{r} d_{p}^{q}:{ }_{r} \mathrm{E}_{p}^{q} \rightarrow{ }_{r} \mathrm{E}_{p-r}^{q-r+1}$ for $r \geq 2$, and ${ }_{2} \mathrm{E}_{p}^{q}=0$ unless $p \geq 0$ and $0 \leq q \leq e$. It follows that $E_{i}=0$ for $i<-q$, and that ${ }_{e+2} \mathrm{E}_{p}^{q}={ }_{\infty} \mathrm{E}_{p}^{q}$.

By Lemma 3 we know that for any fixed $q$ the order of $\left[\operatorname{Tor}_{p}^{R}\left(M, \operatorname{Ext}_{Q}^{q}(N, W)\right)\right]_{0} \in \mathbb{Z}((t))$ goes to infinity together with $p$, hence $\sum_{p}(-1)^{p}\left[{ }_{2} \mathrm{E}_{p}^{q}\right]_{0}$ is in $\mathbb{Z}((t))$. As ${ }_{r} \mathrm{E}_{p}^{q}$ is a subquotient of ${ }_{2} \mathrm{E}_{p}^{q}$, we further have $\sum_{p}(-1)^{p}\left[{ }_{r} \mathrm{E}_{p}^{q}\right]_{0} \in \mathbb{Z}((t))$ for each $r \geq 2$, and we set

$$
\chi\left({ }_{r} \mathrm{E}\right)=\sum_{q=0}^{e}(-1)^{q} \sum_{p}(-1)^{p}\left[{ }_{r} \mathrm{E}_{p}^{q}\right]_{0} .
$$

Next we show that $\chi\left({ }_{r} \mathrm{E}\right)=\chi\left({ }_{r+1} \mathrm{E}\right)$ for $r \geq 2$. To this end, we first note that up to any given degree $n$ each series is determined by a finite number of the summands used to define it, hence when looking at the coefficient of $t^{n}$ we may restrict both summations to the same finite set of indices $p$ and $q$. In $\chi\left({ }_{r} \mathrm{E}\right)$ this coefficient is the Euler characteristic of the degree $n$ part of a finite complex of graded $R$-modules. As the differential ${ }_{r} d_{p}^{q}$ preserves the grading of these modules, the coefficient of $t^{n}$ in $\chi\left({ }_{r+1} \mathrm{E}\right)$ is the Euler characteristic of the degree $n$ part of the homology of this complex. The classical permanence property of Euler characteristics implies that these coefficients are equal.

By the finite convergence of the spectral sequence, we thus see that the series $\chi(E)=$ $\sum_{i}(-1)^{i}\left[E_{i}\right]_{0}$ is equal to $\chi\left({ }_{2} \mathrm{E}\right)$. Together with Lemma 3 this implies

$$
\begin{aligned}
\chi(E) & =\sum_{q=0}^{e}(-1)^{q} \sum_{p}(-1)^{p}\left[\operatorname{Tor}_{p}^{R}\left(M, \operatorname{Ext}_{Q}^{q}(N, W)\right)\right]_{0} \\
& =\sum_{q=0}^{e}(-1)^{q}\left[\chi_{R}\left(M, \operatorname{Ext}_{Q}^{q}(N, W)\right)(t)\right]_{0} \\
& =\left[\frac{H_{M}(t)}{H_{R}(t)} \sum^{e}(-1)^{q} H_{\left.\mathrm{Ext}_{Q}^{q}(N, W)\right)}(t)\right] .
\end{aligned}
$$


In order to finish the computation we use the equality of rational functions

$$
\sum_{q=0}^{e}(-1)^{q} H_{\operatorname{Ext}_{Q}^{q}(A, W)}(t)=(-1)^{e} H_{A}\left(t^{-1}\right)
$$

which holds for each finite $Q$-module $A$. Indeed, if $A=Q(b)$, then the only non-vanishing Ext is $\left.\operatorname{Ext}_{Q}^{0}(Q(b), W)\right) \cong Q\left(-b-\sum_{j=1}^{e} d_{j}\right)$, and the equality is checked by a direct computation which uses the expression $H_{Q}(t)=1 / \prod_{j=1}^{e}\left(1-t^{d_{j}}\right)$. The general case follows, as each $A$ has a finite resolution by finite direct sums of translates of $Q$, and both sides of the formula are additive functions on the Grothendieck group $\mathrm{G}(Q)$ of the category of finite graded $Q$-modules and homomorphisms of degree 0 . Thus, we have established that

$$
\chi(E)=(-1)^{e}\left[\frac{H_{M}(t) \cdot H_{N}\left(t^{-1}\right)}{H_{R}(t)}\right]_{0}=(-1)^{e}\left[\phi_{R}(M, N)\left(t^{-1}\right)\right]_{0} .
$$

Now we turn to the second spectral sequence. It has ${ }^{2} \mathrm{E}^{p, q}=0$ unless $p \geq 0$ and $0 \leq q \leq e$, and differentials ${ }^{r} d^{p, q}:{ }^{r} \mathrm{E}^{p, q} \rightarrow{ }^{r} \mathrm{E}^{p-r+1, q-r}$, hence ${ }^{e+2} \mathrm{E}^{p, q}={ }^{\infty} \mathrm{E}^{p, q}$. Furthermore, our assumption implies that ${ }^{r} \mathrm{E}^{p, q}=0$ for $p \gg 0$, hence for each $r \geq 2$ the sum

$$
\chi\left({ }^{r} \mathrm{E}\right)=\sum_{p}(-1)^{p} \sum_{q=0}^{e}(-1)^{q}\left[{ }^{r} \mathrm{E}^{p, q}\right]_{0}
$$

is actually finite. This time we may apply directly the classical argument on Euler characteristics. In view of formula (2) it yields

$$
\begin{aligned}
\chi(E) & =\sum_{p}(-1)^{p} \sum_{q=0}^{e}(-1)^{q}\left[\operatorname{Ext}_{Q}^{q}\left(\operatorname{Ext}_{R}^{p}(M, N), W\right)\right]_{0} \\
& =\sum_{p}(-1)^{p}\left[\sum_{q=0}^{e}(-1)^{q} H_{\operatorname{Ext}_{Q}^{q}\left(\operatorname{Ext}_{R}^{p}(M, N), W\right)}(t)\right]_{0} \\
& =(-1)^{e}\left[\sum_{p}(-1)^{p} H_{\mathrm{Ext}_{R}^{p}(M, N)}\left(t^{-1}\right)\right]_{0} .
\end{aligned}
$$

The overall result of the preceding computations now reads

$$
\left[\sum_{p}(-1)^{p} H_{\mathrm{Ext}_{R}^{p}(M, N)}\left(t^{-1}\right)\right]_{0}=\left[\phi_{R}(M, N)\left(t^{-1}\right)\right]_{0}
$$

and this clearly implies the desired equality of rational functions.

Proof of Proposition 2. Consider $N^{\vee}=\operatorname{Hom}_{K}(N, K)$ with the induced structure of graded $R$-module. By Lemma 3, the expansion around 0 of the rational function $\chi^{R}(M, N)(t)$ is equal to $\sum_{i}(-1)^{i}\left[\operatorname{Tor}_{i}^{R}\left(M, N^{\vee}\right)\right]_{0} \in \mathbb{Z}((t))$. As $\operatorname{rank}_{K} N$ is finite, we have $H_{N \vee}(t)=$ $H_{N}\left(t^{-1}\right) \in \mathbb{Z}\left[t, t^{-1}\right]$, and thus an equality of formal Laurent series

$$
\sum(-1)^{i}\left[H_{\operatorname{Tor}_{i}^{R}\left(M, N^{\vee}\right)}(t)\right]_{0}=\left[\frac{H_{M}(t) \cdot H_{N}\left(t^{-1}\right)}{H_{R}(t)}\right]_{0}
$$


In the commutative diagram of homomorphisms of rings

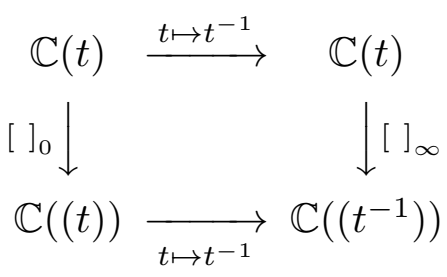

the lower row is an isomorphism of topological fields, hence the preceding equality yields

$$
\sum_{i \geqslant 0}(-1)^{i}\left[H_{\operatorname{Tor}_{i}^{R}\left(M, N^{\vee}\right)}\left(t^{-1}\right)\right]_{\infty}=\left[\frac{H_{M}\left(t^{-1}\right) \cdot H_{N}(t)}{H_{R}\left(t^{-1}\right)}\right]_{\infty} .
$$

The canonical isomorphisms $\left(M \otimes_{R}\left(N^{\vee}\right)\right)^{\vee} \cong \operatorname{Hom}_{R}\left(M, N^{\vee \vee}\right) \cong \operatorname{Hom}_{R}(M, N)$ extend to isomorphisms of graded $R$-modules $\left(\operatorname{Tor}_{i}^{R}\left(M, N^{\vee}\right)\right)^{\vee} \cong \operatorname{Ext}_{R}^{i}(M, N)$ for $i \geq 0$. Thus, in $\mathbb{Z}\left[t, t^{-1}\right]$ we have $H_{\operatorname{Tor}_{i}^{R}\left(M, N^{\vee}\right)}\left(t^{-1}\right)=H_{\operatorname{Ext}_{R}^{i}(M, N)}(t)$, and this finishes the proof.

\section{BASS NUMBERS}

Let $N$ be a finite graded $R$-module. For an integer $i$ and a prime ideal $\mathfrak{p}$ of $R$, the rank of $\operatorname{Ext}_{R_{\mathfrak{p}}}^{i}\left(k(\mathfrak{p}), N_{\mathfrak{p}}\right)$ over the field $k(\mathfrak{p})=R_{\mathfrak{p}} / \mathfrak{p} R_{\mathfrak{p}}$ is known as the $i$ 'th Bass number of $N$ and is denoted $\mu_{R}^{i}(\mathfrak{p}, N)$. We establish nontrivial lower bounds for the Bass numbers $\mu_{R}^{i}(N)=\mu_{R}^{i}\left(R_{+}, N\right)$ of "many" graded modules of finite injective dimension.

A graded ring $R$ is said to be standard if it is generated by elements of degree 1 . In this case $H_{N}(t)$ has a unique expression of the form

$$
H_{N}(t)=\frac{e_{N}(t)}{(1-t)^{n}}
$$

where $n=\operatorname{dim} N$, and $e_{N}(t)$ is a Laurent polynomial in $\mathbb{Z}\left[t, t^{-1}\right]$ such that $e_{N}(1)$ is a positive integer, known as the multiplicity of $N$.

Theorem 4. Let $R$ be a standard graded ring of Krull dimension d.

If $N \neq 0$ is a finite graded $R$-module of finite injective dimension and Krull dimension $n$, then $e_{R}\left(t^{-1}\right)$ divides $e_{N}(t)$ in $\mathbb{Z}\left[t, t^{-1}\right]$. If $p$ is a prime number and $q$ is defined by

$$
q=\max \left\{p^{r} \in \mathbb{Z} \mid \sum_{s=0}^{p^{r}} t^{s} \text { divides } \frac{e_{N}(t)}{e_{R}\left(t^{-1}\right)} \text { in } \mathbb{Z}\left[t, t^{-1}\right]\right\},
$$

then the Bass numbers of $N$ satisfy the inequality

$$
\sum_{i} \mu_{R}^{i}(N) \geq p^{\frac{d-n+q-1}{q(p-1)}}
$$

Proof. Since each $\operatorname{Ext}_{R}^{i}(K, N)$ is a finite graded $K$-vector space, and is trivial for $i>$ $\operatorname{inj} \operatorname{dim}_{R} N$, the rational function $\sum_{i}(-1)^{i} H_{\operatorname{Ext}_{R}^{i}(K, N)}(t)$ is a Laurent polynomial, which we denote by $\phi_{R}^{N}(t)$. Theorem 1 and formula (3) yield an equality in $\mathbb{Z}\left[t, t^{-1}\right]$ :

$$
(1-t)^{d-n} e_{N}(t)=(-t)^{d} e_{R}\left(t^{-1}\right) \phi_{R}^{N}(t) .
$$

As $d \geq n$ and $e_{R}(1) \neq 0$, unique factorization in $\mathbb{Z}\left[t, t^{-1}\right]$ shows that $e_{R}\left(t^{-1}\right)$ divides $e_{N}(t)$. This proves the first assertion. The second one follows by the argument of $[1 ; \S 4]$. 
Corollary 5. If -1 is not a root of the Laurent polynomial $e_{N}(t) / e_{R}\left(t^{-1}\right)$, then

$$
\sum_{i} \mu_{R}^{i}(N) \geq 2^{d-n}
$$

This inequality holds, in particular, when the multiplicity of $N$ is an odd number.

Remark. If $N$ is a finite module over a local $\operatorname{ring}(S, \mathfrak{n})$, and $\operatorname{depth}_{S} N=g<d=\operatorname{dim} S$, then a result of Bruns [4; (9.6.1.a)] implies that $\sum_{i} \mu_{S}^{i}(N) \geq(d-g)(d-g-1)+3$.

The quadratic lower bound above is in general weaker than the exponential bound given by the corollary, so we raise the question whether an inequality $\sum_{i} \mu_{S}^{i}(\mathfrak{n}, N) \geq 2^{d-n}$ holds for each finite $S$-module $N$ of Krull dimension $n$ and of finite injective dimension. Such a bound would be best possible: if $\mathrm{x}$ is a system of parameters of $S, E$ is the injective envelope of $S / \mathfrak{n}$, and $N$ is the finite length module $\operatorname{Hom}_{S}(S /(\mathbf{x}), E)$, then $\mu_{S}^{i}(\mathfrak{n}, N)=\left(\begin{array}{c}d \\ i\end{array}\right)$ since $S$ is Cohen-Macaulay by the Bass Conjecture proved by Peskine, Szpiro, Hochster, and P. Roberts, cf. [4; (9.6.2), (9.6.4.b)].

\section{LAURENT COEFFICIENTS}

We turn to properties of the coefficients of Laurent expansions around 1 of rational functions representing the Hilbert series of finite $R$-modules. For purposes of calibration, it is convenient to write such an expansion in the form

$$
[M]_{1}=\sum_{j \geqslant 0} \frac{f_{R}^{j}(M)}{(1-t)^{d-j}}
$$

with $d$ equal to the Krull dimension of $R$, and to set $f_{R}^{j}(M)=0$ for $j<0$. We call $f_{R}^{j}(M)$ the $j$ 'th Laurent coefficient of $M$ and note that it is a rational number.

Remark. When $R$ is standard and $d-\operatorname{dim} M=h$, comparison with (3) yields $\operatorname{rank}_{K}\left(M_{n}\right)=$ $\sum_{j=h}^{d-1} f_{R}^{j}(M)\left(\begin{array}{c}n+d-j-1 \\ d-j-1\end{array}\right)$ for $n \gg 0$, hence the Laurent coefficients of the principal part of $[M]_{1}$ are directly related to the coefficients of the Hilbert polynomial of $M$ in the usual "binomial" format, as in $[4 ;(4.1 .5)]: f_{R}^{j}(M)=(-1)^{h-j} e_{h-j}(M)$ for $h \leq j \leq d-1$.

Under the assumptions of Theorem 1, the $j$ 'th coefficient in the Laurent expansion around 1 of the rational function $\phi_{R}(M, N)(t)$ is an alternating sum of the corresponding Laurent coefficients of Ext modules. The number

$$
\varepsilon_{R}^{j}(M, N)=\sum_{i=0}^{j}(-1)^{i} f_{R}^{j}\left(\operatorname{Ext}_{R}^{i}(M, N)\right)
$$

represents a portion of such a sum. The next result shows that under a homological finiteness hypothesis it contains the entire information.

Proposition 6. If the projective dimension of a finite graded $R$-module $M$ or the injective dimension of a finite graded $R$-module $N$ is finite, then

$$
\left[\phi_{R}(M, N)(t)\right]_{1}=\sum \frac{\varepsilon_{R}^{j}(M, N)}{(1-t)^{d-j}} .
$$


The proposition applies to all finite modules over a graded polynomial ring $R$, and raises the question of extending its validity - at least on part - to other graded rings. When

$$
\phi_{R}(M, N)(t)=\sum_{j=0}^{n} \frac{\varepsilon_{R}^{j}(M, N)}{(1-t)^{d-j}}+O\left(\frac{1}{(1-t)^{d-n-1}}\right)
$$

for some integer $n \geq 0$, we say that the sequences $\phi_{R}^{*}(M, N)$ (of coefficients of the Laurent expansion of $\phi_{R}(M, N)(t)$ around 1) and $\varepsilon_{R}^{*}(M, N)=\left\{\varepsilon_{R}^{j}(M, N)\right\}_{j \in \mathbb{Z}}$ agree up to level $n$.

The next result shows that an agreement of $\varepsilon^{*}$ and $\phi^{*}$ imposes strong restrictions on the local structure of $R$. We adopt the convention that the codimension of a prime ideal $\mathfrak{p}$ of $R$ is the non-negative integer $\operatorname{codim} \mathfrak{p}=\operatorname{dim} R-\operatorname{dim}(R / \mathfrak{p})$ and note that height $\mathfrak{p} \leq \operatorname{codim} \mathfrak{p}$, with equality when $R$ is an integral domain. The $\operatorname{ring} R$ is regular in codimension $c$ if $R_{\mathfrak{p}}$ is regular for each prime $\mathfrak{p}$ such that codim $\mathfrak{p} \leq c$; by Matijevic [8; 2.1$)]$ it suffices to impose the condition only on the primes in Proj $R$, the set of homogeneous prime ideals of $R$.

Proposition 7. Let $\mathfrak{p}$ be a homogeneous prime ideal of $R$ with codim $\mathfrak{p}=h$. The sequences $\varepsilon_{R}^{*}(R / \mathfrak{p}, R / \mathfrak{p})$ and $\phi_{R}^{*}(R / \mathfrak{p}, R / \mathfrak{p})$ agree up to level $h$ precisely when the local ring $R_{\mathfrak{p}}$ is regular, and if furthermore $h=0$ then $\mathfrak{p}$ is the unique codimension 0 prime of $R$.

The condition that $\varepsilon_{R}^{*}(M, N)$ and $\phi_{R}^{*}(M, N)$ agree up to level $n$ can be rewritten as

$$
H_{R}\left(t^{-1}\right) \cdot \sum_{j=0}^{n} \frac{\varepsilon_{R}^{j}(M, N)}{(1-t)^{d-j}}=H_{M}\left(t^{-1}\right) \cdot H_{N}(t)+O\left(\frac{1}{(1-t)^{2 d-n-1}}\right) .
$$

Taking on both sides Laurent expansions around 1, we can further reformulate it in terms of a system of $n+1$ numerical equalities involving the numbers $f_{R}^{j}(M), f_{R}^{j}(N), f_{R}^{j}(R)$, and $\varepsilon_{R}^{j}(M, N)$ for $0 \leq j \leq n$. Equations (4.0), (4.1), (4.2) display such a system for $n=2$.

Theorem 8. Let $M$ and $N$ be finite graded modules over a graded ring $R$.

If $R$ has a unique prime $\mathfrak{r}$ of codimension 0 and $R_{\mathfrak{r}}$ is a field, then

$$
f_{R}^{0}(R) \varepsilon_{R}^{0}(M, N)=f_{R}^{0}(M) f_{R}^{0}(N) .
$$

If $R$ is an integral domain which is regular in codimension 1 , then

$$
f_{R}^{0}(R) \varepsilon_{R}^{1}(M, N)-f_{R}^{1}(R) \varepsilon_{R}^{0}(M, N)=f_{R}^{0}(M) f_{R}^{1}(N)-f_{R}^{1}(M) f_{R}^{0}(N) .
$$

If $R$ is a unique factorization domain which is regular in codimension 2 , then

$$
\begin{gathered}
f_{R}^{0}(R) \varepsilon_{R}^{2}(M, N)-f_{R}^{1}(R) \varepsilon_{R}^{1}(M, N)+\left(f_{R}^{2}(R)-f_{R}^{1}(R)\right) \varepsilon_{R}^{0}(M, N)= \\
f_{R}^{0}(M) f_{R}^{2}(N)-f_{R}^{1}(M) f_{R}^{1}(N)+\left(f_{R}^{2}(M)-f_{R}^{1}(M)\right) f_{R}^{0}(N) .
\end{gathered}
$$

Remark. When $R$ is a domain for any finite graded $R$-module $M$ one has

$$
f_{R}^{0}(M)=\operatorname{rank}_{R}(M) f_{R}^{0}(R),
$$

cf. Lemma 10(c) below. If furthermore $R$ is regular in codimension 1, then formula (1), due to Benson and Crawley-Boevey $[3 ;(2.4)]$, is seen to follow from (4.0) and (4.1).

Recall that a graded complete intersection is a quotient of a graded polynomial ring by a regular sequence of homogeneous elements. By a classical result of Grothendieck [7; (XI.3.14)], cf. also [5], a complete intersection which is regular in codimension 3 is factorial, 
Corollary 9. If $R$ is a graded complete intersection which is regular in codimension 3, then the equalities of the theorem hold for all finite graded $R$-modules.

In the next section we show that neither the UFD hypothesis of the theorem nor the codimension 3 hypothesis of the corollary can be significantly weakened. The proofs of the results above depend on a few lemmas; the first one (for $\psi=f_{R}^{1}$ ) is in $[3 ;(2.2),(2.3)]$.

Lemma 10. Let $M \neq 0$ be a finite $R$-modules with $h=\operatorname{dim} R-\operatorname{dim} M$.

(a) $f_{R}^{j}(-)$ is an additive function on $\mathrm{G}(R)$ for each $j \in \mathbb{Z}$.

(b) $f_{R}^{j}(M)=0$ for $j<h$ and $f_{R}^{h}(M)>0$.

(c) $f_{R}^{h}(M)=\sum_{\substack{\mathfrak{p} \in \operatorname{Proj} R \\ \text { codim } \mathfrak{p}=h}} \operatorname{length}_{R_{\mathfrak{p}}}\left(M_{\mathfrak{p}}\right) f_{R}^{h}(R / \mathfrak{p})$.

Proof. (a) results from the additivity of []$_{1}$ and the uniqueness of Laurent expansions.

(b) is a fact of dimension theory.

(c) is a formal consequence of the preceding two because $M$ admits a finite filtration with subquotients of the form $(R / \mathfrak{q})(a)$ for appropriate $\mathfrak{q} \in \operatorname{Proj} R$ and $a \in \mathbb{Z}$.

Lemma 11. Let $M$ and $N$ be finite $R$-modules, such that for some $c \in \mathbb{N}$ and for all $\mathfrak{p} \in \operatorname{Proj} R$ with codim $\mathfrak{p} \leq c$ either $\operatorname{proj} \operatorname{dim}_{R_{\mathfrak{p}}}\left(M_{\mathfrak{p}}\right)$ or $\operatorname{inj} \operatorname{dim}_{R_{\mathfrak{p}}}\left(N_{\mathfrak{p}}\right)$ is finite. For each integer $j \leq c$ and any submodule $L$ of $\operatorname{Ext}_{R}^{i}(M, N)$, we have $f_{R}^{j}(L)=0$ when $i>j$.

Proof. By Lemma 10(b) it suffices to show that $j<\operatorname{dim} R-\operatorname{dim} L=g$, hence we may assume that $g \leq c$. If $\mathfrak{p} \in \operatorname{Supp} L$ is a homogeneous prime with codim $\mathfrak{p}=g$, then

$$
\operatorname{Ext}_{R_{\mathfrak{p}}}^{i}\left(M_{\mathfrak{p}}, N_{\mathfrak{p}}\right) \cong \operatorname{Ext}_{R}^{i}(M, N)_{\mathfrak{p}} \supseteq L_{\mathfrak{p}} \neq 0
$$

implies that $i \leq \min \left\{\operatorname{proj} \operatorname{dim}_{R_{\mathfrak{p}}}\left(M_{\mathfrak{p}}\right)\right.$, inj $\left.\operatorname{dim}_{R_{\mathfrak{p}}}\left(N_{\mathfrak{p}}\right)\right\}$. One of these dimensions is finite by assumption, and thus is bounded above by depth $R_{\mathfrak{p}}$, cf. [4; (1.3.3) and (3.1.17)]. Thus, we have $j<i \leq \operatorname{depth} R_{\mathfrak{p}} \leq \operatorname{codim} \mathfrak{p}=g$, as desired.

Proof of Proposition 6: Equate the Laurent expansions around 1 of the rational functions in Theorem 1 and use Lemma 11 to regroup the terms in the sum of Ext's.

The argument for part (a) of the next lemma is abstracted from the proof of $[3 ;(2.4)]$.

Lemma 12. The numbers $\varepsilon_{R}^{j}(M, N)$ have the following properties.

(a) $\varepsilon_{R}^{j}(-,-)$ is a biadditive function on $\mathrm{G}(R)$ if $R$ is regular in codimension $c$ and $j \leq c$.

(b) $\varepsilon_{R}^{j}(M, N)=0$ when $j<\operatorname{dim} R-\operatorname{dim}(R /(\operatorname{ann} M+\operatorname{ann} N))$.

Proof. (a) Fix an integer $j$ between 0 and $c$, take a short exact sequence of finite $R$-modules $0 \rightarrow N^{\prime} \rightarrow N \rightarrow N^{\prime \prime} \rightarrow 0$, and consider the exact sequence

$$
\begin{aligned}
& 0 \rightarrow \operatorname{Hom}_{R}\left(M, N^{\prime}\right) \rightarrow \operatorname{Hom}_{R}(M, N) \rightarrow \operatorname{Hom}_{R}\left(M, N^{\prime \prime}\right) \rightarrow \\
& \operatorname{Ext}_{R}^{1}\left(M, N^{\prime}\right) \rightarrow \quad \cdots \quad \rightarrow \operatorname{Ext}_{R}^{j-1}\left(M, N^{\prime \prime}\right) \rightarrow \\
& \operatorname{Ext}_{R}^{j}\left(M, N^{\prime}\right) \rightarrow \operatorname{Ext}_{R}^{j}(M, N) \rightarrow \operatorname{Ext}_{R}^{j}\left(M, N^{\prime \prime}\right) \rightarrow N^{j+1} \rightarrow 0 .
\end{aligned}
$$

By assumption, the ring $R_{\mathfrak{p}}$ has global dimension $\leq j$ whenever codim $\mathfrak{p} \leq c$, hence Lemma 11 shows that $f_{R}^{j}\left(N^{j+1}\right)=0$. The additivity of $\varepsilon_{R}^{j}(M,-)$ now follows by applying $f_{R}^{j}$ to the long exact sequence. The additivity of $\varepsilon_{R}^{j}(-, N)$ yields to a similar approach.

(b) For each $i$ the $R$-module $\operatorname{Ext}_{R}^{i}(M, N)$ is annihilated by ( $\left.\operatorname{ann} M+\operatorname{ann} N\right)$, hence we have $\operatorname{dim} R-\operatorname{dim} \operatorname{Ext}_{R}^{i}(M, N) \geq \operatorname{dim} R-\operatorname{dim} R /(\operatorname{ann} M+\operatorname{ann} N)>j$, and thus $\varepsilon_{R}^{j}(M, N)$ 
Proof of Proposition 7. Lemma 10(b) shows that $f_{R}^{h}(R / \mathfrak{p})$ and $f_{R}^{0}(R)$ are positive, and that

$$
\left[\phi_{R}(R / \mathfrak{p}, R / \mathfrak{p})(t)\right]_{1}=(-1)^{h} \cdot \frac{f_{R}^{h}(R / \mathfrak{p})}{f_{R}^{0}(R)} \cdot \frac{f_{R}^{h}(R / \mathfrak{p})}{(1-t)^{d-2 h}}+O\left(\frac{1}{(1-t)^{d-2 h-1}}\right)
$$

With $\eta_{h}(\mathfrak{p})=\sum_{i=0}^{h}(-1)^{i} \operatorname{rank}_{k(\mathfrak{p})}\left(\operatorname{Ext}_{R_{\mathfrak{p}}}^{i}(k(\mathfrak{p}), k(\mathfrak{p}))\right)$, Lemmas 12(b) and 10(c) give

$$
\sum_{j=0}^{h} \frac{\varepsilon_{R}^{j}(R / \mathfrak{p}, R / \mathfrak{p})}{(1-t)^{d-j}}=\eta_{h}(\mathfrak{p}) \cdot \frac{f_{R}^{h}(R / \mathfrak{p})}{(1-t)^{d-h}}
$$

Thus, $\varepsilon_{R}^{*}(R / \mathfrak{p}, R / \mathfrak{p})$ and $\phi_{R}^{*}(R / \mathfrak{p}, R / \mathfrak{p})$ agree up to level 0 if and only if $f_{R}^{0}(R)=$ $f_{R}^{0}(R / \mathfrak{p})$. In view of Lemma $10(\mathrm{c})$, this is equivalent to the statement that $\mathfrak{p}$ is the only prime of $R$ with codim $\mathfrak{p}=0$ and that $R_{\mathfrak{p}}$ is a field.

If $h>0$, then $\varepsilon_{R}^{*}(R / \mathfrak{p}, R / \mathfrak{p})$ and $\phi_{R}^{*}(R / \mathfrak{p}, R / \mathfrak{p})$ agree up to level $h$ if and only if $\eta_{h}(\mathfrak{p})=0$. When the local ring $R_{\mathfrak{p}}$ is regular the rank of $\operatorname{Ext}_{R_{\mathfrak{p}}}^{i}(k(\mathfrak{p}), k(\mathfrak{p}))$ over $k(\mathfrak{p})$ is equal to $\left(\begin{array}{c}h \\ i\end{array}\right)$, hence $\eta_{h}(\mathfrak{p})=0$. When $R_{\mathfrak{p}}$ is not regular its embedding dimension is at least 2 because $\operatorname{dim} R_{\mathfrak{p}}=h \geq 1$, and then $\eta_{h}(\mathfrak{p}) \neq 0$ by Okiyama [9; Proposition 8].

Lemma 13. (a) If $M$ and $N$ are finite graded $R$-modules, then for $a, b \in \mathbb{Z}$ the sequences $\varepsilon_{R}^{*}(M(a), N(b))$ and $\phi_{R}^{*}(M(a), N(b))$ agree up to level $n$ if and only if $\varepsilon_{R}^{*}(M, N)$ and $\phi_{R}^{*}(M, N)$ agree up to the same level.

(b) If $\mathfrak{p}$ is a homogeneous prime ideal such that codim $\mathfrak{p}=h$ and the local ring $R_{\mathfrak{p}}$ is Gorenstein, then $\varepsilon_{R}^{*}(R / \mathfrak{p}, R)$ and $\phi_{R}^{*}(R / \mathfrak{p}, R)$ agree up to level $h$.

Proof. (a) The canonical isomorphisms $\operatorname{Ext}_{R}^{i}(M(a), N(b)) \cong \operatorname{Ext}_{R}^{i}(M, N)(b-a)$ show that

$$
\sum_{j=0}^{n} \frac{\varepsilon_{R}^{j}(M(a), N(b))}{(1-t)^{d-j}}=t^{a-b} \cdot \sum_{j=0}^{n} \frac{\varepsilon_{R}^{j}(M, N)}{(1-t)^{d-j}}
$$

holds for each $n \geq 0$, while it is clear that $\phi_{R}(M(a), N(b))(t)=t^{a-b} \cdot \phi_{R}(M, N)(t)$.

(b) By a direct computation as in the proof of Proposition 7, we get

$$
\left[\phi_{R}(R / \mathfrak{p}, R)(t)\right]_{1}=(-1)^{h} \cdot \frac{f_{R}^{h}(R / \mathfrak{p})}{(1-t)^{d-h}}+O\left(\frac{1}{(1-t)^{d-h-1}}\right)
$$

On the other hand, the Gorenstein hypothesis yields $\mu_{R}^{i}(\mathfrak{p}, R)=\delta_{i h}$, hence

$$
\sum_{j=0}^{h} \frac{\varepsilon_{R}^{j}(R / \mathfrak{p}, R)}{(1-t)^{d-j}}=(-1)^{h} \cdot \frac{f_{R}^{h}(R / \mathfrak{p})}{(1-t)^{d-h}}
$$


Proof of Theorem 8. Due to Lemmas 10(a) and 12(a), both sides of (4.n) are biadditive functions on $\mathrm{G}(R)$ for $n=0,1,2$. Thus, it suffices to consider $M$ and $N$ which are translates of quotients of $R$ by homogeneous prime ideals. By Lemma 13(a), we can even assume that $M=R / \mathfrak{p}$ and $N=R / \mathfrak{q}$ with $\mathfrak{p}, \mathfrak{q} \in \operatorname{Proj} R$.

If $\operatorname{codim} \mathfrak{p}>n$, or $\operatorname{codim} \mathfrak{q}>n$, or $\operatorname{codim} \mathfrak{p}=n=\operatorname{codim} \mathfrak{q}$ and $\mathfrak{p} \neq \mathfrak{q}$, then the left hand side of (4.n) vanishes by Lemma 12(b), and its right hand side vanishes by Lemma 10(b). If codim $\mathfrak{p}=n$ and $\mathfrak{p}=\mathfrak{q}$ (respectively, if $\mathfrak{p}=0$ or if $\mathfrak{q}=0$ ), then (4. $n$ ) holds by Proposition 7 (respectively, by Proposition 6 or by Lemma 13(b)).

It remains to prove (4.2) when $\operatorname{codim} \mathfrak{s}=1$, where $\mathfrak{s}=\mathfrak{p}$ or $\mathfrak{s}=\mathfrak{q}$. As $R$ is factorial, $\mathfrak{s}=(x)$ for a homogeneous $x \in R$, hence by additivity and Lemma 13(a) we get the result from the exact sequence $0 \rightarrow R(-\operatorname{deg} x) \rightarrow R \rightarrow R / \mathfrak{s} \rightarrow 0$ and already settled cases.

Remark 14. Let $M$ be a finite graded module over a graded integral domain $R$.

If $R$ is Gorenstein in codimension 1, then

$$
f_{R}^{1}\left(\operatorname{Hom}_{R}(M, R)\right)-f_{R}^{1}\left(\operatorname{Ext}_{R}^{1}(M, R)\right)=2 f_{R}^{1}(R) \operatorname{rank}_{R}(M)-f_{R}^{1}(M) .
$$

If $R$ is factorial, then in addition

$$
\begin{gathered}
f_{R}^{2}\left(\operatorname{Hom}_{R}(M, R)\right)-f_{R}^{2}\left(\operatorname{Ext}_{R}^{1}(M, R)\right)+f_{R}^{2}\left(\operatorname{Ext}_{R}^{2}(M, R)\right)= \\
\left(1+2 \frac{f_{R}^{1}(R)}{f_{R}^{0}(R)}\right)\left(f_{R}^{1}(R) \operatorname{rank}_{R}(M)-f_{R}^{1}(M)\right)+f_{R}^{2}(M) .
\end{gathered}
$$

Indeed, the formulas above are obtained by rewriting (4.1) and (4.2) for $N=R$ with the help of (5). A factorial domain is Cohen-Macaulay in codimension 2 (due to normality), and thus is Gorenstein in this codimension by a result of Murthy, cf. [4; (3.3.19)]. The argument for Lemma $12\left(\right.$ a) shows that $\varepsilon_{R}^{j}(-, R)$ is an additive function on $\mathrm{G}(R)$ for $j=0,1,2$. At this point we may retrace the preceding proof, ignoring any discussion of primes $\mathfrak{q} \neq 0$.

\section{NORMAL DOMAINS}

Our examples are built from the ring $R=K[X, Y, U, V] /(X V-Y U)=K[x, y, u, v]$ with the standard grading, and the $R$-module $M=R /(u, v) \cong K[X, Y]$.

Example 15. Equality (4.2) may fail over a 3-dimensional graded hypersurface ring $R$ which is regular in codimension 2 (and hence is a normal domain).

Over $K[X, Y, U, V]$ the quadratic form $X V-Y U$ has a homogeneous matrix factorization

$$
\left(\begin{array}{cc}
V & -Y \\
-U & X
\end{array}\right)\left(\begin{array}{cc}
X & Y \\
U & V
\end{array}\right)=\left(\begin{array}{cc}
X V-Y U & 0 \\
0 & X V-Y U
\end{array}\right)=\left(\begin{array}{cc}
X & Y \\
U & V
\end{array}\right)\left(\begin{array}{cc}
V & -Y \\
-U & X
\end{array}\right)
$$

hence Eisenbud $[6 ;(4.1)]$ provides the following minimal graded free resolution of $M$ :

$\ldots \rightarrow R^{2}(-4) \stackrel{\left(\begin{array}{cc}v & -y \\ -u & x\end{array}\right)}{\longrightarrow} R^{2}(-3) \stackrel{\left(\begin{array}{ll}x & y \\ u & v\end{array}\right)}{\longrightarrow} R^{2}(-2) \stackrel{\left(\begin{array}{cc}v & -y \\ -u & x\end{array}\right)}{\longrightarrow} R^{2}(-1) \stackrel{(u, v)}{\longrightarrow} R \rightarrow 0$. 
Applying to it the functor $\operatorname{Hom}_{R}(-, M)$ we get a complex of graded $R$-modules

$$
0 \rightarrow M \stackrel{0}{\rightarrow} M^{2}(1) \stackrel{\left(\begin{array}{cc}
0 & 0 \\
-y & x
\end{array}\right)}{\longrightarrow} M^{2}(2) \stackrel{\left(\begin{array}{ll}
x & 0 \\
y & 0
\end{array}\right)}{\longrightarrow} M^{2}(3) \stackrel{\left(\begin{array}{cc}
0 & 0 \\
-y & x
\end{array}\right)}{\longrightarrow} M^{2}(4) \rightarrow \ldots
$$

Direct computations based on the $M$-regularity of the sequence $x, y$ yield

$$
\begin{gathered}
M \cong \operatorname{Hom}_{R}(M, M) \cong \operatorname{Ext}_{R}^{1}(M, M) \\
\operatorname{Ext}_{R}^{2 i}(M, M) \cong k(2 i) \quad \text { for } i \geq 1 \\
\operatorname{Ext}_{R}^{2 i+1}(M, M)=0 \quad \text { for } i \geq 1
\end{gathered}
$$

Accordingly, we get Laurent expansions

$$
\begin{gathered}
H_{R}(t)=\frac{1-t^{2}}{(1-t)^{4}}=\frac{2}{(1-t)^{3}}-\frac{1}{(1-t)^{2}} ; \\
H_{M}(t)=H_{\operatorname{Hom}_{R}(M, M)}(t)=H_{\operatorname{Ext}_{R}^{1}(M, M)}(t)=\frac{1}{(1-t)^{2}} ; \\
H_{\operatorname{Ext}_{R}^{2}(M, M)}(t)=\frac{1}{t^{2}}=\sum_{j \geqslant 0}(j+1)(1-t)^{j} .
\end{gathered}
$$

It follows that for $M=N$ the expression on the left hand side of (4.2) is equal to 0 , while the one on its right hand side is equal to -1 .

Example 16. Equality (6.2) may fail over a 4-dimensional graded Cohen-Macaulay ring $R^{\prime}$ of type 2 which is regular in codimension 3 (and hence is a normal domain).

Let $R^{\prime}=R[Z, W] /(x W-u Z, y W-v Z)=K[x, y, z, u, v, w]$ with the standard grading, and set $M^{\prime}=R^{\prime} /(u, v, w) \cong K[X, Y, Z]$. Since $w$ is $R^{\prime}$-regular and annihilates $M^{\prime}$, for $S=R^{\prime} /(w) \cong R[Z] /(u Z, v Z)$ and for each $i$ we have $\operatorname{Ext}_{R^{\prime}}^{i}\left(M^{\prime}, R^{\prime}\right) \cong \operatorname{Ext}_{S}^{i-1}\left(M^{\prime}, S\right)(1)$. As $M^{\prime}=M[Z]$, and the minimal free resolution of $M[Z]$ over $R[Z]$ is "the same as" that of $M$ over $R$, it is easy to get a beginning of a minimal $S$-free resolution of $M^{\prime}$ in the form

$$
S^{4}(-2) \stackrel{\left(\begin{array}{cccc}
v & -y & z & 0 \\
-u & x & 0 & z
\end{array}\right)}{\longrightarrow} S^{2}(-1) \stackrel{(u, v)}{\longrightarrow} S \rightarrow 0
$$

Thus, we are interested in the homology of the complex

$$
0 \rightarrow S \stackrel{\alpha=\left(\begin{array}{l}
u \\
v
\end{array}\right)}{\longrightarrow} S^{2}(1) \stackrel{\beta=\left(\begin{array}{cc}
v & -u \\
-y & x \\
z & 0 \\
0 & z
\end{array}\right)}{\longrightarrow} S^{4}(2)
$$

In degree 0 we have $\operatorname{Ker} \alpha=(0: S(u, v))=z S \cong M^{\prime}(-1)$, hence $\operatorname{Ext}_{R^{\prime}}^{1}\left(M^{\prime}, R^{\prime}\right) \cong M^{\prime}$.

In degree 1 the complex is exact, hence $\operatorname{Ext}_{R^{\prime}}^{2}\left(M^{\prime}, R^{\prime}\right)=0$. Indeed, writing $b \in S^{2}(1)$ in the form $\left(r_{1}+s_{1} z, r_{2}+s_{2} z\right)$ with uniquely determined $r_{j} \in R$, we see that $\beta(b)=0$ implies 
From the last two equations we get

$$
s_{j} \in\left(0: S z^{2}\right)=\left((0: S z):_{S} z\right)=\left((u, v):_{S} z\right)=(u, v) S
$$

hence $b=\left(r_{1}, r_{2}\right)$. In view of the preceding example, the first two equations yield an $a \in R$ such that $r_{1}=a u$ and $r_{2}=a v$, hence $b=a(u, v) \in \operatorname{Im} \alpha$.

We can now collect the relevant data on Laurent expansions:

$$
\begin{gathered}
H_{R^{\prime}}(t)=\frac{1-3 t^{2}+2 t^{3}}{(1-t)^{6}}=\frac{3}{(1-t)^{4}}-\frac{2}{(1-t)^{3}} ; \\
H_{M^{\prime}}(t)=H_{\mathrm{Ext}_{R^{\prime}}^{1}\left(M^{\prime}, R^{\prime}\right)}(t)=\frac{1}{(1-t)^{3}} ; \\
H_{\mathrm{Hom}_{R^{\prime}}\left(M^{\prime}, R^{\prime}\right)}(t)=H_{\mathrm{Ext}_{R^{\prime}}{ }^{\prime}\left(M^{\prime}, R^{\prime}\right)}(t)=0 .
\end{gathered}
$$

They show that the left hand side of (6.2) is equal to 0 , and its right hand side to $\frac{1}{3}$.

\section{REFERENCES}

1. Avramov, L. L. and Buchweitz, R.-O., Lower bounds for Betti numbers, Compositio Math. 86 (1993), $147-158$.

2. Avramov, L. L. and Foxby, H.-B., Homological dimensions of unbounded complexes, J. Pure. Appl. Algebra 71 (1991), 129-155.

3. Benson, D. J. and Crawley-Boevey, W. W., A ramification formula for Poincaré series, and a hyperplane formula for modular invariants, Bull. London Math. Soc. (to appear).

4. Bruns, W. and Herzog, J., Cohen-Macaulay rings, Cambridge Stud. Adv. Math., vol. 39, Univ. Press, Cambridge, 1993.

5. Call., F. and Lyubeznik, G., A simple proof of Grothendieck's theorem on the parafactoriality of local rings, Birational algebra, syzygies, and multiplicities (W. J. Heinzer, C. L. Huneke, J. D. Sally, eds.), Contemp. Math., vol. 159, Amer. Math. Soc., Providence, RI, 1994, pp. 15-18.

6. Eisenbud, D., Homological algebra on a complete intersection, with an application to group representations, Trans. Amer. Math. Soc. 260 (1980), 35-64.

7. Grothendieck, A., Cohomologie locale des faisceaux cohérents et théorèmes de Lefschetz locaux et globaux (SGA 2), Adv. Stud. Pure Math., vol. 2, North-Holland, Amsterdam, 1968.

8. Matijevic, J., Three local conditions on a graded ring, Trans. Amer. Math. Soc. 205 (1975), 275-284.

9. Okiyama, S., A local ring is CM if and only if its residue field has a CM syzygy, Tokyo J. Math. 14 (1991), 489-500.

Department of Mathematics, Purdue University, West Lafayette, Indiana 47907, U. S. A. E-mail address: avramov@math.purdue.edu

Department of Mathematics, University of Toronto, Toronto, Ontario M5S 1A1, Canada E-mail address: ragnar@lake.scar.utoronto.ca

Department of Mathematics, Northwestern University, Evanston, Illinois 60208, U. S. A. E-mail address: judy@math.nwu.edu 